\title{
Fiber-based high-dimensional quantum key distribution with twisted photons
}

Cozzolino, Daniele; Bacco, Davide; Lio, Beatrice Da; Ingerslev, Kasper; Ding, Yunhong; Dalgaard, Kjeld; Kristensen, Poul; Galili, Michael; Rottwitt, Karsten; Ramachandran, Siddharth

Total number of authors:

11

Published in:

Conference on Lasers and Electro-Optics/Pacific Rim, CLEOPR 2018

Link to article, DOI:

10.1364/CLEOPR.2018.Th5A.1

Publication date:

2018

Document Version

Peer reviewed version

Link back to DTU Orbit

Citation (APA):

Cozzolino, D., Bacco, D., Lio, B. D., Ingerslev, K., Ding, Y., Dalgaard, K., Kristensen, P., Galili, M., Rottwitt, K., Ramachandran, S., \& Oxenløwe, L. K. (2018). Fiber-based high-dimensional quantum key distribution with twisted photons. In Conference on Lasers and Electro-Optics/Pacific Rim, CLEOPR 2018 (Vol. Part F113CLEOPR 2018). [Paper Th5A.1] IEEE. https://doi.org/10.1364/CLEOPR.2018.Th5A.1

\section{General rights}

Copyright and moral rights for the publications made accessible in the public portal are retained by the authors and/or other copyright owners and it is a condition of accessing publications that users recognise and abide by the legal requirements associated with these rights.

- Users may download and print one copy of any publication from the public portal for the purpose of private study or research.

- You may not further distribute the material or use it for any profit-making activity or commercial gain

- You may freely distribute the URL identifying the publication in the public portal 


\title{
Fiber based high-dimensional quantum communication with twisted photons
}

\author{
Daniele Cozzolino ${ }^{* 1}$, Davide Bacco ${ }^{+1}$, Beatrice Da Lio ${ }^{1}$, Kasper Ingerslev ${ }^{1}$, Yunhong Ding ${ }^{1}$, Kjeld Dalgaard ${ }^{1}$, \\ Poul Kristensen ${ }^{2}$, Michael Galili ${ }^{1}$, Karsten Rottwitt ${ }^{1}$, Siddharth Ramachandran ${ }^{3}$, Leif Katsuo Oxenløwe ${ }^{1}$ \\ ${ }^{1}$ CoE SPOC, DTU Fotonik, Dep. Photonics Eng., Technical University of Denmark, Orsteds Plads 340, Kgs. Lyngby, 2800 Denmark \\ 2 OFS-Fitel, Priorparken 680, DK-2605, Broendby, Denmark \\ ${ }^{3}$ Electrical and Computer Engineering Department, Boston University, 8 St Mary's St, Boston, MA, USA \\ Emails: *dacoz@fotonik.dtu.dk; ${ }^{\dagger}$ dabac@fotonik.dtu.dk \\ Dated: March 28, 2018
}

\begin{abstract}
Quantum communication (QC) concerns the faithful transmission of quantum states between separated parties, and represents a key enabler for many quantum applications, ranging from quantum key distribution $(\mathrm{QKD})$ to distributed quantum computing. However, low information rates and short propagation distances, due to high channel losses and perturbations of the quantum states, limit the development of this field and its practical applications. Highdimensional (Hi-D) quantum communication can address these challenges by adding more information per photon, directly enhancing the information rate and the system's error tolerance. We transmit Hi-D quantum states using photons prepared in four orbital angular momentum (OAM) modes in a $1.2 \mathrm{~km}$ long OAM-carrying fiber. This is the first experimental demonstration of Hi-D quantum states transmission using the OAM basis over an optical fiber. Furthermore, we successfully implement a real-time decoy-state Hi-D QKD protocol, and demonstrate the highest secret key rate and longest transmission distance of OAM-QKD presented to date.
\end{abstract}

\section{Introduction}

Quantum communication $(\mathrm{QC})$, i.e. the transfer of a quantum state from one user to another, is an emerging field with many promising applications [1] 2]. Its usefulness and range of applicability strongly depends on the extent to which quantum channels can be reliably and efficiently implemented. The distribution of quantum states may be obtained through optical channels like optical fibers, free-space links or indeed under-water links [2]-8]. The main limitations in QC experiments today are the relatively short achievable transmission distances (a few hundred $\mathrm{km}$ ) and the low transmittable bit rates. Introducing high-dimensional (Hi-D) quantum states, it is possible to increase the information rate per transmitted photon, as each photon will carry more information. This higher information efficiency has the benefit of increasing the robustness to channel noise, resulting in an increased error threshold [9]-11]. The generation, transmission and detection of high-dimensional quantum states is very challenging and only a few experimental realizations have been achieved for Hi-D QC protocols [12-15|. Multiple degrees of freedom, or a combination of them, can be used for the generation of $\mathrm{Hi}$ D-quantum states, for instance time, frequency, space, phase and polarization [2, 16]. However, only some of these can be considered unbounded and are not limiting the final performance of the system. For example, by increasing the dimensions in the time-encoding scheme, the repetition rate of the system is lowered, so the overall information rate does not increase, whereas frequency- and space-encoding are theoretically unbounded enabling increased information rates (see Supp. Mat.).
Spatial states, like lateral modes, constitute a suitable discrete set of Hi-D states for quantum encoding. Orbital angular momentum (OAM) modes are particularly interesting for fiber based communications, since their orthogonality is well preserved (low crosstalk) through an optical fiber. These modes are characterized by an azimuthal phase dependence $(\exp (i \ell \theta))$ that generates a twisted wavefront, and are composed by photons carrying $\ell h$ of orbital angular momentum, where $\ell$ is an integer referred to as the topological charge. However, OAM fiber transmission with more than two modes has only been utilized for classical communication so far [17, 18|. Quantum and classical communications with twisted light have been demonstrated up to $143 \mathrm{~km}$ [19-26]. In this work, we experimentally demonstrate the first transmission of Hi-D quantum states, encoded in four OAM modes and their superposition, over a $1.2 \mathrm{~km}$ long OAM-supporting fiber. To verify the correct transmission of the quantum states, we implement both a two-dimensional (2D) and a $4 \mathrm{D}$ decoy state quantum key distribution protocol [27]. Figure 1 c) shows a comparison to hitherto published space-encoding QKD works. This work presents the highest transmitted key rate and the longest transmission distance to date for OAM systems.

\section{High-dimensional quantum states using OAM}

The quantum states used in this work are based on a sub-space of four OAM states designated by their topological charges $\Omega=\{|+6\rangle,|-6\rangle,|+7\rangle,|-7\rangle\}$ and supported by the OAM fiber (Figure $1 \mathrm{~b}$ )). To generate and detect these OAM states 

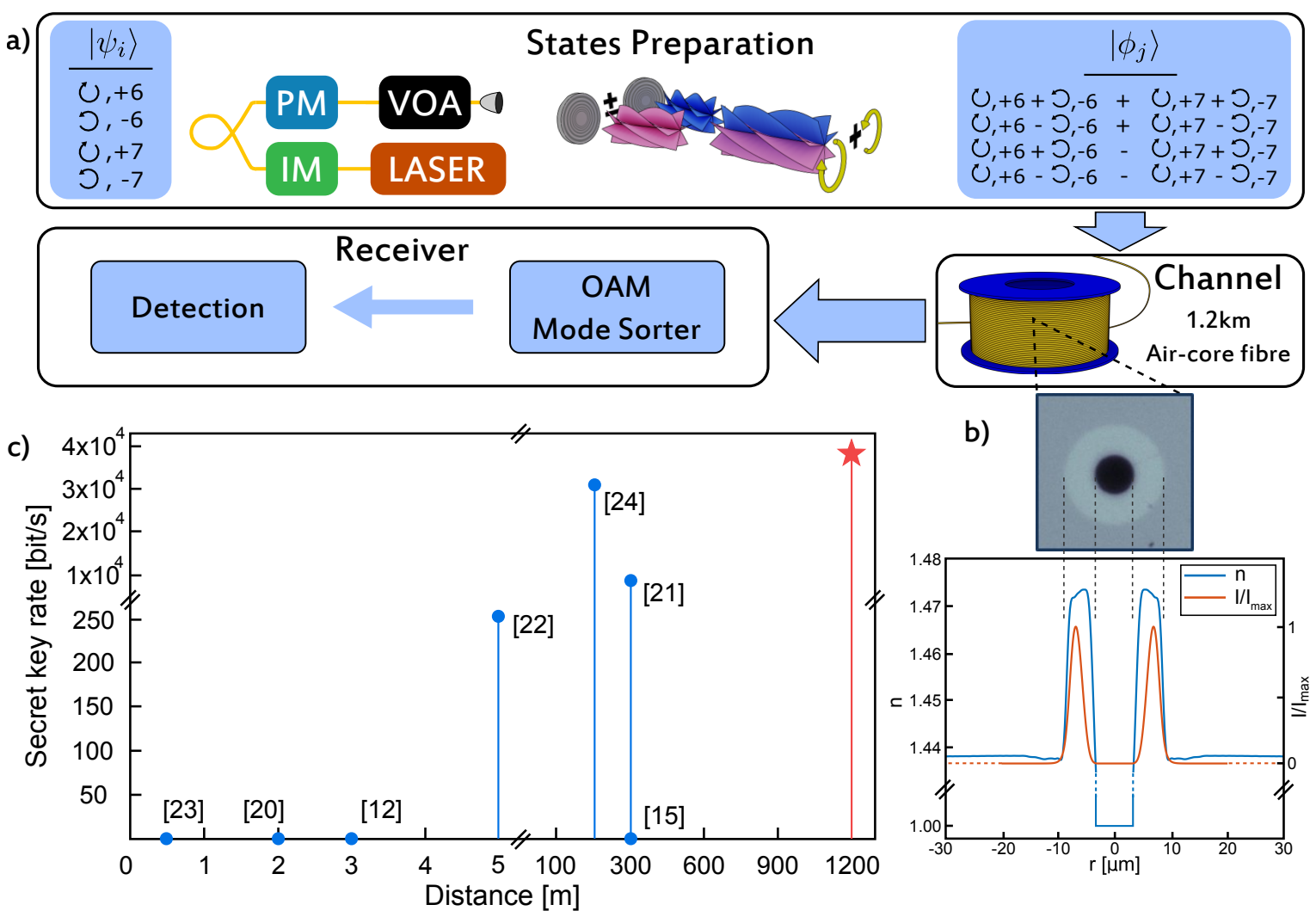

Figure 1 a) Schematic of the experiment. A continuous wave (CW) laser beam is carved into pulses using an intensity modulator (IM). A phase modulator (PM) is used to actively control the polarization of the pulses and a variable optical attenuator (VOA) is utilized to reach the quantum regime. The weak coherent pulses, with polarization states $|L\rangle,|R\rangle,|D\rangle,|A\rangle(\circlearrowleft \equiv|L\rangle$ and $\circlearrowright \equiv|R\rangle)$, are sent through vortex plates to obtain an OAM charge (determined by the input polarization) and are then ready for the transmission through the air-core OAM-carrying fiber. After fiber transmission, the weak pulses are sorted according to their topological charge magnitude and detected. The quantum states prepared are $\left|\psi_{i}\right\rangle$ (left-side) and $\left|\phi_{j}\right\rangle$ (right-side), where the states $\left|\phi_{j}\right\rangle$ in figure are not normalized. b) Air-core fiber. The air-core fiber cross-section is shown along with the refractive index profile $(n)$. The $3 \mu \mathrm{m}$ radius air gap in the middle creates a large index contrast to a high-index ring (radius $8.25 \mu \mathrm{m}$ ), which facilitates the propagation of OAM modes with their characteristic circular intensity patterns $\left(I / I_{\max }\right)$. c) Performance comparison. The demonstrated key rate $\left(R_{s k}\right)$ as a function of distance for different space-encoding based QC experiments is reported. Blue dots are demonstrations reported in literature. Red star is the secret key rate obtained in this work for a fiber based $4 D$ QKD protocol. Note that [12, 15, 20, 22, 24] are real-time implementations of QC and [21] is a proof of principle experiment. This work and references $[12,15]$ are performed at telecom wavelengths, i.e. around $1550 \mathrm{~nm}$.

in practice, we furthermore employ the polarization degree of freedom. Our generic quantum states can thus be written as:

$$
|\Theta\rangle=\sum_{n=1}^{D} a_{n}|\sigma, \ell\rangle
$$

where $\sum_{n=1}^{D}\left|a_{n}\right|^{2}=1, D$ is the Hilbert space dimension and $|\sigma\rangle$ and $|\ell\rangle$ are the polarization and OAM quantum states respectively. We adopt the standard basis for the polarization states as $\Pi=\{|L\rangle,|R\rangle\}$ (left- and right-handed circular polarizations), so that diagonal and anti-diagonal states can be written as $|D\rangle=(|R\rangle+|L\rangle) / \sqrt{2}$ and $|A\rangle=(|R\rangle-|L\rangle) / \sqrt{2}$, respectively. Thus, in Equation $11, D=8$ and $|\sigma, \ell\rangle=$ $|\sigma\rangle_{\Pi}|\ell\rangle_{\Omega}$, with $\sum_{n=1}^{8}\left|a_{n}\right|^{2}=1$. In our implementation, we use only quantum states whose spin angular momentum (SAM) (i.e. the polarization) and OAM are anti-aligned. States with aligned SAM and OAM can in principle be used as well, but for simplicity of the experimental setup, we confined the experiment to the anti-aligned case. As a consequence, the Hilbert space shrinks from an eight- to a four-dimensional space. Hence, our general ququart is given by:

$$
|\Theta\rangle=a_{1}|R,+6\rangle+a_{2}|L,-6\rangle+a_{3}|R,+7\rangle+a_{4}|L,-7\rangle
$$

with $\sum_{k=1}^{4}\left|a_{k}\right|^{2}=1$. We now define the states $\left|\psi_{i}\right\rangle$ and $\left|\phi_{j}\right\rangle$ (with $i, j=1, \ldots, 4$ ), which constitute the two mutually unbiased bases (MUBs) for the Hi-D QKD application. The states $\left|\psi_{i}\right\rangle$ constitute the four-dimensional computational basis (Figure 1 a) left), whereas the states $\left|\phi_{j}\right\rangle$, corresponding to superpositions of $\left|\psi_{i}\right\rangle$, are given by the expression $\frac{1}{2} \sum_{m=1}^{4} \mathrm{e}^{i \zeta_{m}}|\sigma, \ell\rangle_{m}$ with $\zeta_{m} \in\{0, \pi\}$ (Figure 1 a) right). See Supp. Mat.. 


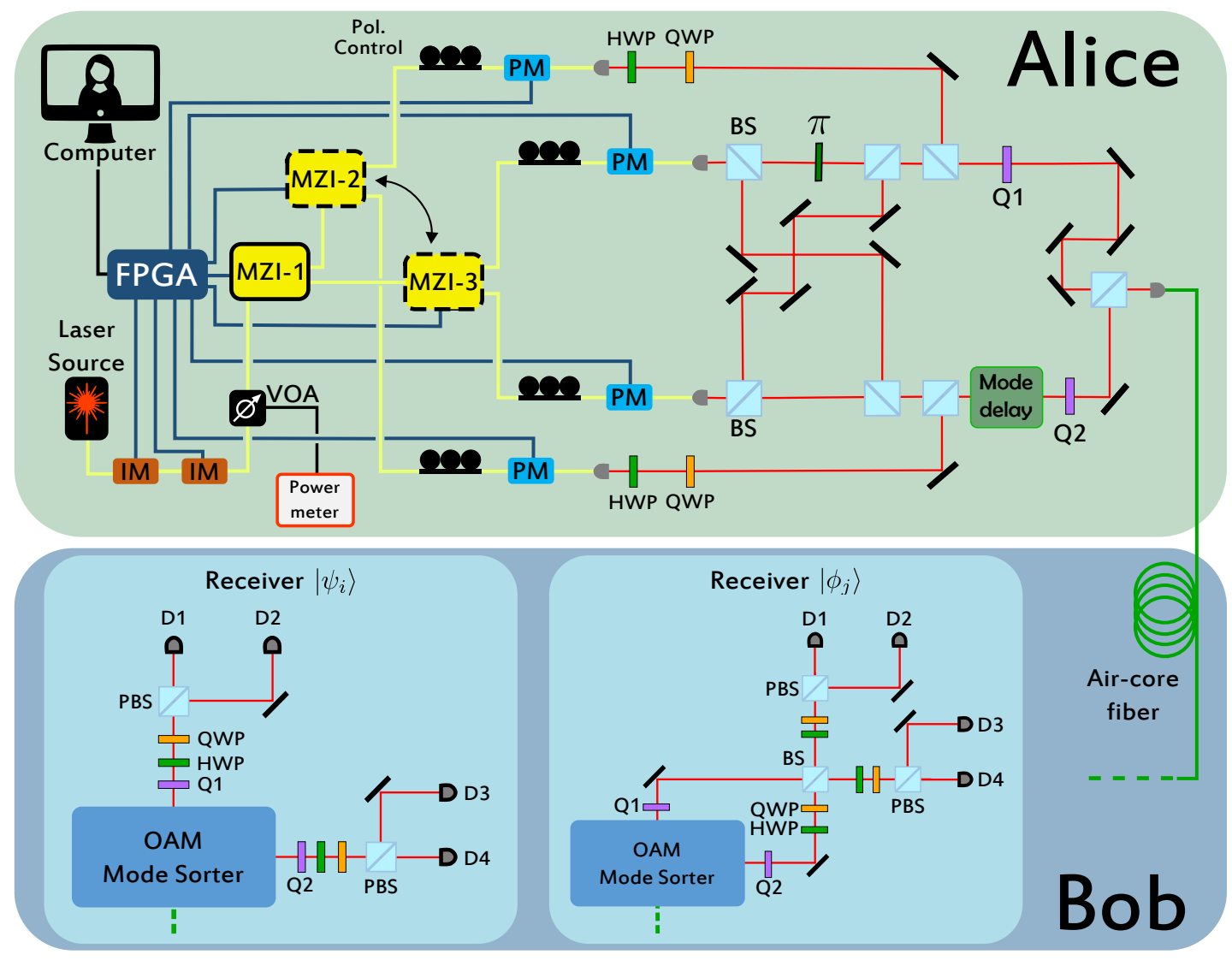

Figure 2 Experimental setup. Fast Mach-Zehnder Interferometer (MZI) switches, controlled by digital signals from a field programmable gate array (FPGA), allow the real-time preparation of $\left|\psi_{i}\right\rangle$ and $\left|\phi_{j}\right\rangle$ states. Only a single MZI is required to separately generate the states $\left|\psi_{i}\right\rangle$ and $\left|\phi_{j}\right\rangle$ (dashed edges MZI). Note that, within the set $\left|\psi_{i}\right\rangle$ or $\left|\phi_{j}\right\rangle$, the states are prepared in real-time mode. At the input of the MZI we inject a continuous wave laser $(1550 \mathrm{~nm}$ ) carved into pulses by an intensity modulator (IM) driven by the same FPGA. A second IM is used to implement a three-intensity decoy-state technique for the QKD protocols. A variable optical attenuator (VOA) allows to reach the quantum regime. The polarization of the quantum states is prepared by a PM. Two different voltages are chosen to yield D and A polarizations at the output of the PM. Vortex plates with topological charge $\ell=+6$ and $\ell=+7(\mathrm{Q} 1, \mathrm{Q} 2)$ assign different OAM orders to the quantum states. The quantum states are then coupled into the air-core fiber. After the fiber transmission, an OAM mode sorter is implemented to separate even from odd modes $(\ell=|6|$ and $\ell=|7|)$. Two other vortex plates, identical to the previous, are used to convert from OAM to Gaussian modes. After the OAM sorter, the quantum states $\left|\psi_{i}\right\rangle$ and $\left|\phi_{j}\right\rangle$ are separated using their polarization. HWP and QWP are used to convert the circular to linear polarization, while a polarization beam splitter (PBS) is used to separate between two orthogonal polarizations. To measure the relative phase between different angular momenta ( $|\ell|$-values) in the $\left|\phi_{j}\right\rangle$ states, a free-space MZI is used at the output of the mode sorter. Four single photon detectors (D1-D4) are connected to the outputs. The insertion loss is measured to be approximately $9 \mathrm{~dB}$ for the $\left|\psi_{i}\right\rangle$ receiver and $10 \mathrm{~dB}$ for the $\left|\phi_{j}\right\rangle$ receiver.

\section{Generation of the quantum states}

Figure 1 a) shows a schematic of the experiment and the basic concept is described in the caption text. Essentially, weak coherent pulses are polarization modulated through a phase modulator (PM) and subsequently vortex plates allows for the spin-orbit coupling. 4D OAM symbols are thus created, transmitted and detected. Figure 2 shows the detailed experimental setup. An intensity modulator (IM), controlled by a field programmable gate array (FPGA), carves out pulses with 0.6 $\mathrm{GHz}$ repetition rate and $100 \mathrm{ps}$ pulse width of a continuous wave $(\mathrm{CW})$ laser emitting at $1550 \mathrm{~nm}$. A variable optical attenuator (VOA) is used to reach the quantum regime (in average less than one photon per-pulse). Fast Mach-Zehnder interferometers (MZIs) allow real-time preparation and selection of the quantum states $\left|\psi_{i}\right\rangle$ and $\left|\phi_{j}\right\rangle$. MZI-1 is used to select between the two Hi-D MUBs, where MZI-2 is used to encode states from $\left|\psi_{i}\right\rangle$ and MZI-3 is used to encode from $\left|\phi_{j}\right\rangle$. For this experiment, only one MZI is employed, allowing the realtime preparation of the quantum states $\left|\psi_{i}\right\rangle$ or $\left|\phi_{j}\right\rangle$, i.e. one MUB at time. Note that we are not limiting the performance of the real-time QKD protocol, since the two MUBs are exclusive, i.e. they are never selected at the same time. Moreover, for QKD protocols a second IM is used to implement a threeintensity decoy-state technique [27]. The MZI chooses a path through the transmitter, which corresponds to the generation of specific OAM states. In each path a PM modulates the polarization of the weak coherent pulse. These polarizations determine the OAM state when passed through the vortex-plates 

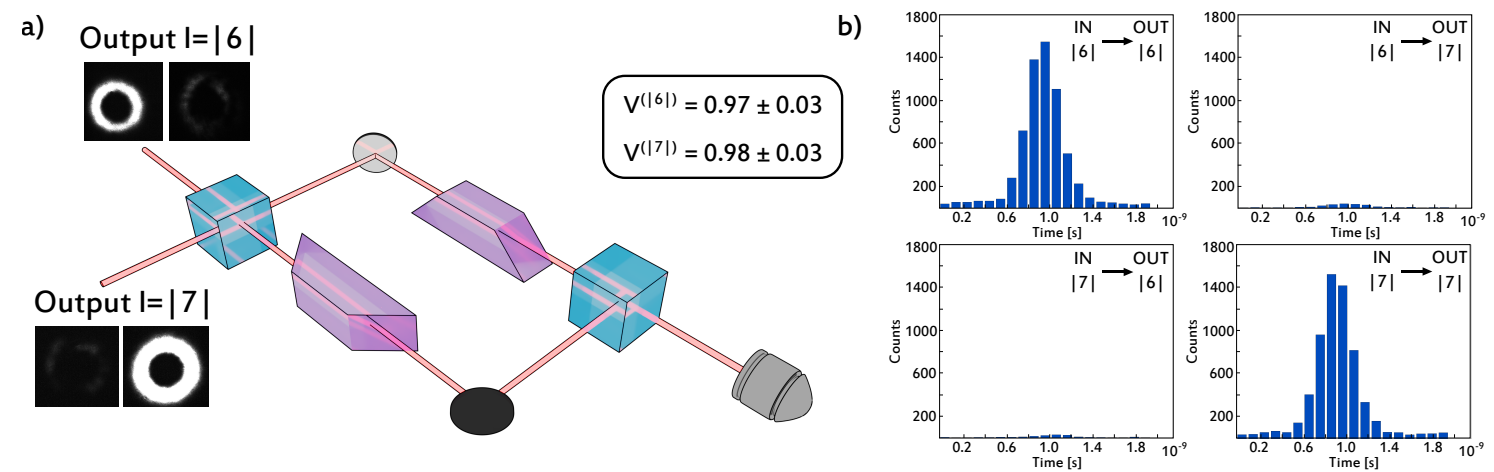

Figure 3 a) Schematic of the OAM mode sorter and its performance with classical light. Implementation of the OAM sorter, which divides even from odd spatial modes. In our case the sorter is used to split $\ell=|6|$ from $\ell=|7|$. A continuous wave laser at $1550 \mathrm{~nm}$ is used to characterize the OAM sorter with classical light, and the output intensity profiles for the input $\ell=|6|$ and $\ell=|7|$ are shown. Images are acquired with an infrared camera. We select one single $|\ell|$ order as input and the two outputs are simultaneously captured (ER of $19 \mathrm{~dB})$. b) Performance of the mode sorter with weak coherent pulses. A weak pulsed laser (mean photon number per pulse $\left.\mu=(5.2 \pm 0.2) \times 10^{-3}\right)$ at $1550 \mathrm{~nm}$, with pulse width $100 \mathrm{ps}$ and repetition rate of $0.6 \mathrm{GHz}$, is used to test the mode sorter in the quantum regime after the transmission of the quantum states through $1.2 \mathrm{~km}$ fiber. The histograms represent the OAM pulses, folded on a time-slot, for the different input/output combinations. The visibilities obtained in the quantum regime are $V^{(|6|)}=0.97 \pm 0.03$ and $V^{(|7|)}=0.98 \pm 0.03$.

(Q1, Q2). Following the central path, via MZI-3, two specific voltage settings of the PMs are used to generate the diagonal (D) and anti-diagonal (A) polarizations at the output of the PMs, which subsequently are used to create the $\left|\phi_{j}\right\rangle$ states. To create the $\left|\psi_{i}\right\rangle$ states (MZI-2), free-space half- and quarterwave plates (HWPs, QWPs) are used to obtain left- and righthanded circularly polarized light $(\mathrm{L}, \mathrm{R})$.

The two vortex plates, with topological charge $\ell=+6$ and $\ell=+7$, generate the OAM quantum states $|+6\rangle,|-6\rangle,|+7\rangle$ and $|-7\rangle|28|$. An L-polarized beam acquires a $+\ell$ OAM charge with $\mathrm{R}$ polarization when passing through the vortex plate. Like wise, an R-polarized beam yields a $-\ell$ OAM charge with L polarization. A superposition among OAM states with same $|\ell|$, is obtained by letting photons, with polarization states $|D\rangle$ or $|A\rangle$, pass through the vortex plate corresponding to that $|\ell|$. The superposition of quantum states with different $|\ell|$ is obtained by combining the states || 6|\rangle and || 7|\rangle with beam-splitters. Due to different mode group velocities in the air-core fiber, a free-space delay (about $4.5 \mathrm{~m}$ ) is implemented before coupling the weak pulses to the fiber. This delay is required in order to pre-compensate the different time-of-arrivals of the modes with $\ell=|6|$ and $\ell=|7|$. The quantum states are coupled to the air-core fiber using a $6 \mathrm{~mm}$ aspherical lens mounted on a 3-axis stage. The overall insertion and coupling losses attributed to the transmitter (Alice) are approximately $8 \mathrm{~dB}$.

\section{Air-core fiber as a quantum channel}

The quantum states are coupled to the fiber that enables conservation of orbital angular momentum modes, as depicted in Figure 1 b). The fiber has an air core surrounded by a highrefractive index ring, creating a large index-difference between the center of the fiber and its outer part [29]. This structure splits the spin-orbit aligned and spin-orbit anti-aligned states in effective index, thus allowing transmission of OAM modes with preserved orthogonality. As previously mentioned, we choose only anti-aligned states. The mode delay between $\ell=|6|$ and $\ell=|7|$ is about $15 \mathrm{~ns}$ and the extinction ratio (ER) among them is reported in Table 1 A high extinction ratio is translated into a high purity of the modes and a low crosstalk between them. The measured ER are higher than $18 \mathrm{~dB}$ for both cases, enabling faithful transmission of the states in the fiber. Note that crosstalk stemming from the coupling optics adds to the intrinsic fiber crosstalk. The fiber loss is around $1 \mathrm{~dB} / \mathrm{km}$ at $1550 \mathrm{~nm}$. Characterization of the fiber is reported in Supp. Mat..

Table 1 Modes extinction ratio [dB]. Measured extinction ratio between the modes $\ell=|6|$ and $\ell=|7|$ with SAM and OAM antialigned.

\begin{tabular}{|c|c|c|}
\hline Detected mode & $\ell=|6|$ & $\ell=|7|$ \\
\hline ER & 18.4 & 18.7 \\
\hline
\end{tabular}

\section{Detection of OAM states}

To detect the OAM quantum states the receiver (Bob) implements projective measurements to recover the encoded information. An OAM mode sorter is used to separate even and odd modes, in our case $\ell=|6|$ and $\ell=|7|$, being insensitive to the mode sign. The implemented mode sorter is a free-space MZI with two Dove prisms with a relative angle of $90^{\circ}$ [30]. In Figure 3 a) a schematic of the mode sorter is depicted. In Figure 3 a) the output intensity profiles for the input $\ell=|6|$ and $\ell=|7|$ are reported, showing a large and clear contrast ( $\sim 19 \mathrm{~dB}$ extinction ratio). Figure $3 \mathrm{~b}$ ) shows the results for weak coherent pulses, and the obtained quantum visibilities are $V^{(|6|)}=0.97 \pm 0.03$ and $V^{(|7|)}=0.98 \pm 0.03$. The visibility is defined as $V=\left(n_{\max }-n_{\min }\right) /\left(n_{\max }+n_{\min }\right)$ 

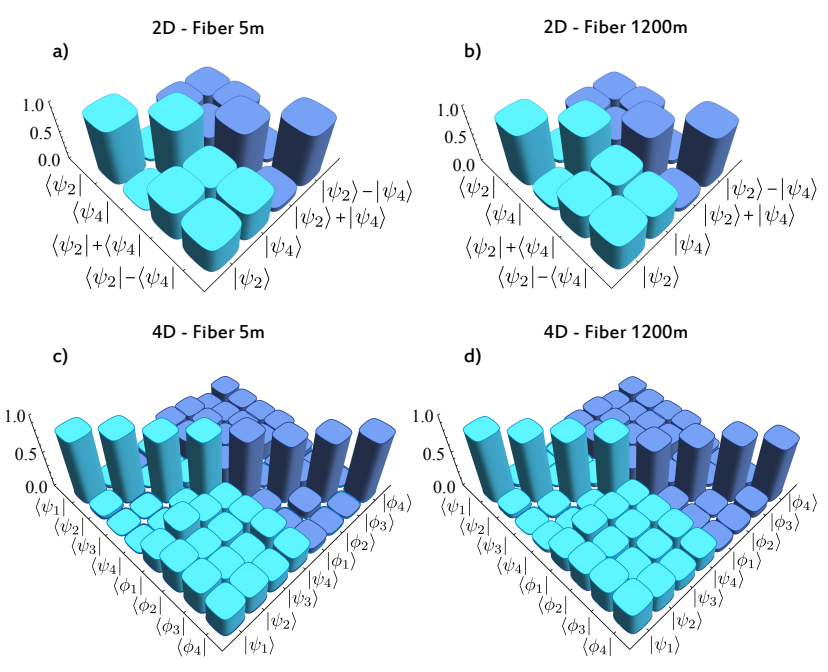

Figure 4 a) 2D tomography measurement with $5 \mathrm{~m}$ fiber. The average fidelity measured over 2 minutes is $0.985 \pm 0.006$. b) $2 \mathrm{D}$ tomography measurement with $1.2 \mathrm{~km}$ fiber. The average fidelity measured over 2 minutes is $0.980 \pm 0.002$. c) $4 \mathrm{D}$ tomography measurement with $5 \mathrm{~m}$ fiber. The average fidelity measured over 2 minutes is $0.963 \pm 0.005$. d) $4 \mathrm{D}$ tomography measurement with $1.2 \mathrm{~km}$ fiber. The average fidelity measured over 5 minutes is $0.954 \pm 0.004$. All measurements were acquired with a mean photon number value of $\mu=(4.4 \pm 0.3) \times 10^{-3}$ for the $5 \mathrm{~m}$ long fiber and $\mu=(9.9 \pm 0.2) \times 10^{-3}$ for the $1.2 \mathrm{~km}$ long fiber.

with $n_{\max }\left(n_{\min }\right)$ the maximum (minimum) number of detected events. Note that the mode sorter is polarization dependent, so QWPs and HWPs are used to compensate polarization misalignments [31]. After the sorting process, the photons are converted back to the fundamental Gaussian mode with two other vortex plates and then conveniently separated according to their polarization. In particular, to measure the quantum states $\left|\psi_{i}\right\rangle$, HWPs, QWPs and two polarization beam-splitters (PBS) are adopted in the two arms of the sorter, as reported in Figure 2 (receiver $\left|\psi_{i}\right\rangle$ ). In the case of $\left|\phi_{j}\right\rangle$ states, a free-space MZI is required to measure the relative phase difference between the OAM modes, as shown in Figure 2 (receiver $\left|\phi_{j}\right\rangle$ ). The photons are then detected by four InGaAs single photon detectors and registered by a time-tagger unit. The insertion loss attributed to Bob's detection system is measured to be 9 $\mathrm{dB}$ in the receiver $\left|\psi_{i}\right\rangle$ and $10 \mathrm{~dB}$ in receiver $\left|\phi_{j}\right\rangle$ (from the output of the OAM fiber to the input of the detectors). These losses may be further decreased by using non-interferometric mode sorters [32, 33]. The entire system is firstly characterized and tested with a $5 \mathrm{~m}$ fiber link. Subsequently, a 1.2 $\mathrm{km}$ fiber is installed for the final experiments and demonstrations. In order to characterize the system, a quantum state tomography technique is implemented for dimension $D=2$ and $D=4$. Figure 4 shows the four matrices for the two fibers, measured with weak laser pulses and mean photon numbers $(\mu)$ of $(4.4 \pm 0.3) \times 10^{-3}$ and $(9.9 \pm 0.2) \times 10^{-3}$ for the $5 \mathrm{~m}$ and $1.2 \mathrm{~km}$, respectively. Using the definition of fidelity $F(p, q)=\sum_{i}\left(p_{i} q_{i}\right)^{1 / 2}$, where $p(q)$ is a discrete proba- bility distribution with elements $p_{i}\left(q_{i}\right)$, we measure in average $0.985 \pm 0.006$ and $0.980 \pm 0.002$ for the qubit $(D=2)$ and $0.963 \pm 0.005$ and $0.954 \pm 0.004$ in the ququart case.

\section{Hi-D quantum key distribution}

To demonstrate the usefulness of quantum communication through the air-core fiber using OAM states, we implement real-time $2 \mathrm{D}$ and $4 \mathrm{D}$ quantum key distribution protocols 34 . 35]. The mutually unbiased bases for the $2 \mathrm{D}$ protocol are obtained by selecting and combining states within the $\left|\psi_{i}\right\rangle$ ensemble. We choose:

$$
\tilde{\mathcal{M}}_{0}=\left(\begin{array}{l}
|L,-6\rangle \\
|L,-7\rangle
\end{array}\right) \text { and } \tilde{\mathcal{M}}_{1}=\frac{1}{\sqrt{2}}\left(\begin{array}{l}
|L,-6\rangle+|L,-7\rangle \\
|L,-6\rangle-|L,-7\rangle
\end{array}\right)
$$

The entire set of $\left|\psi_{i}\right\rangle$ and $\left|\phi_{j}\right\rangle$ states constitute the MUBs for the $4 \mathrm{D}$ protocol, that is:

$$
\begin{gathered}
\mathcal{M}_{0}=\left(\begin{array}{l}
|R,+6\rangle \\
|L,-6\rangle \\
|R,+7\rangle \\
|L,-7\rangle
\end{array}\right) \\
\mathcal{M}_{1}=\frac{1}{2}\left(\begin{array}{l}
|R,+6\rangle+|L,-6\rangle+|R,+7\rangle+|L,-7\rangle \\
|R,+6\rangle-|L,-6\rangle+|R,+7\rangle-|L,-7\rangle \\
|R,+6\rangle+|L,-6\rangle-|R,+7\rangle+|L,-7\rangle \\
|R,+6\rangle-|L,-6\rangle-|R,+7\rangle-|L,-7\rangle
\end{array}\right)
\end{gathered}
$$

Following the standard authentication process between Alice and Bob, Alice randomly switches between the two MUBs and modulates the secret key on the different states (two or four, depending on the dimension). Usually, one of the two bases provides the key and the other monitors the presence of an eavesdropper. After the transmission through the OAM-fiber, Bob decides in which of the two bases to project the quantum states (i.e. $\mathcal{M}_{0}\left(\tilde{\mathcal{M}}_{0}\right)$ or $\mathcal{M}_{1}\left(\tilde{\mathcal{M}}_{1}\right)$ bases). Photons measured in the wrong basis will be discarded during the sifting procedure. The protocol implemented can be considered as a BB84 with a three-intensity decoy-state method $(\mu, v, \omega)$ with dimensions $D=2$ and $D=4$. After the transmission of the quantum states, the error correction and the privacy amplification procedures are implemented. The final secret key rate is thus established using [35]:

$$
R \geq I_{A B}-\min \left(I_{A E}, I_{B E}\right)
$$

where $I_{A B}$ represents the classical mutual information between Alice and Bob $\left(I_{X Y}=h(X)-h(X \mid Y)\right)$, with the marginal entropy defined as $h(X)=\sum_{x \in X}-p(x) \log _{2} p(x)$, where $p(x)$ is the marginal probability distribution. The final secret key rate in Equation (5) depends on the strategy of the eavesdropper. We here consider the case of individual and collective attacks (IAs, CAs) [35|. In the case of decoy-state quantum key distribution the secret key rate is [11]:

$$
R_{s k}=Q_{\psi, 1}\left[\log _{2} D-h_{H i-D}\left\{e_{\phi, 1}\right\}\right]-Q_{\psi} \Delta_{l e a k}
$$



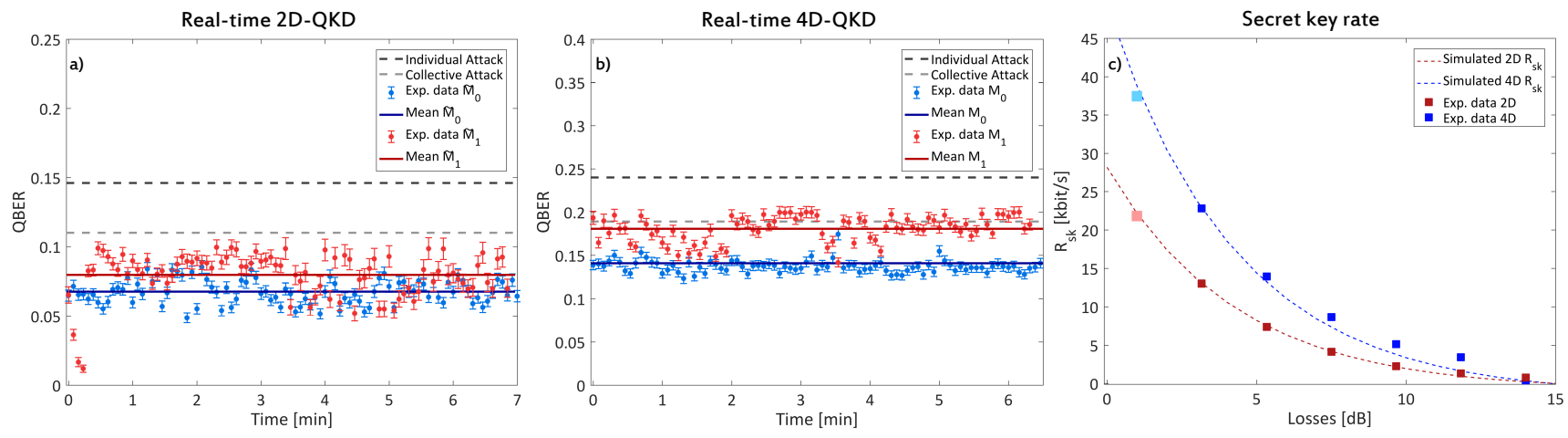

Figure 5 a) Real-time QBER as a function of time for the qubit encoding. Blue dots are the experimental data collected in the $\tilde{\mathcal{M}}_{0}$ basis. Red dots represent data for the $\tilde{\mathcal{M}}_{1}$ basis. The average QBERs in the $\tilde{\mathcal{M}}_{0}$ and $\tilde{\mathcal{M}}_{1}$ bases are $6.7 \%$ and $7.9 \%$ respectively. Both QBER values are below the 2D individual attacks (IAs) and collective attacks (CAs) limit, that is $14.6 \%$ and $11.0 \%$ respectively. b) Real-time QBER as a function of time for the ququart encoding. Blue dots are the experimental data collected in the $\mathcal{M}_{0}$ basis. Red ones represent data for the $\mathcal{M}_{1}$ basis. The average QBERs in the $\mathcal{M}_{0}$ and $\mathcal{M}_{1}$ bases are $14.1 \%$ and $18.1 \%$ respectively. Both QBER values are below the high dimensional IAs and CAs thresholds, that is $24.0 \%$ and $18.9 \%$ respectively. c) Transmitted secret key rate as a function of the channel losses. The light blue (light red) square represents the rate measured after transmission through the $1.2 \mathrm{~km}$ fiber, while the blue (red) squares are obtained by adding further channel losses with a variable optical attenuator in the case $\mathrm{D}=4(\mathrm{D}=2)$. The secret key rates obtained after $1.2 \mathrm{~km}$ long fiber are $21.8 \mathrm{kHz}$ for $D=2$ and $37.43 \mathrm{kHz}$ for $D=4$. The parameters used are $\mu=(9.9 \pm 0.2) \times 10^{-3}, v=(8.8 \pm 0.1) \times 10^{-3}, \omega=3.2 \times 10^{-7}$, detectors efficiency $\eta_{d}=0.2$ and detectors dead time $t_{d}=20 \mu \mathrm{s}$.

Here $Q_{\psi, 1}$ and $Q_{\psi}$ are the single photon and the overall gain in the $\mathcal{M}_{0}$ or $\tilde{\mathcal{M}}_{0}$ basis and $e_{\phi, 1}$ is the single photon error rate in the $\mathcal{M}_{1}$ or $\tilde{\mathcal{M}}_{1}$ bases. The gain, $Q_{z}$ with $z$ number of photons, is defined as the ratio between Bob's detection events and Alice's number of emitted pulses. $h_{H i-D}(x)$ is the binary entropy in the $D$-dimensional case, $\Delta_{\text {leak }}=h_{H i-D}\left(e_{\phi}\right)$ is the fraction of the key revealed during the error correction, and $e_{\phi}$ is the overall error rate in $\mathcal{M}_{1}$ or $\tilde{\mathcal{M}}_{1}$ bases. The single-photon statistics are estimated with the three-intensity decoy state technique. Here we assume that Alice and Bob exchange an infinitelylong key for simplicity, although a finite-key scenario could equally well be considered for the key extraction [36, 37]. In our experimental demonstration (in the case $D=4$ ), we use a relatively new result that shows that it is possible to obtain a complete unconditional security proof by using fewer states in the check basis [37]. In our case we prepare the complete $\mathcal{M}_{0}$ basis for key generation, and $\left|\phi_{1,2,3}\right\rangle$ for the $\mathcal{M}_{1}$ basis. Further analysis of the secret key rate is reported in the Supp. Mat..

\section{Results and discussion}

The results reported in Figue 5 a) and b) show a complete demonstration of the $2 \mathrm{D}$ and $4 \mathrm{D}$ real-time quantum communication protocol. Average QBER values of $6.7 \%$ in the $\tilde{\mathcal{M}}_{0}$ basis and $7.9 \%$ in the $\tilde{\mathcal{M}}_{1}$ basis are measured. Furthermore, in the case of high-dimensional encoding we measured an average QBER of $14.1 \%$ in the $\mathcal{M}_{0}$ basis and $18.1 \%$ in $\mathcal{M}_{1}$. The QBER values are obtained over $\sim 7$ minutes of measurement time and the average QBERs are below the individual and collective attack thresholds. In Figure 5 c) we show the experimental secret key rate for $D=2$ and $D=4$ as a function of the channel loss. The light blue and light red squares are the experimental data measured after the propagation through 1.2 $\mathrm{km}$ fiber. Red and blue squares are data obtained by adding further channel loss with a VOA. The experimental data fit the theoretical prediction (dashed lines), showing that the secret key extraction would be guaranteed by our protocol over a distance of $\sim 14 \mathrm{~km}$, with a secret key rate of $0.78 \mathrm{kHz}$ and 0.40 $\mathrm{kHz}$, for the $2 \mathrm{D}$ and $4 \mathrm{D}$ case respectively. Positive secret key rates of $21.81 \mathrm{kHz}(D=2)$ and $37.43 \mathrm{kHz}(D=4)$ are obtained after $1.2 \mathrm{~km}$ fiber. An enhancement of $71 \%$ in the final key rate is achieved by using high-dimensional encoding. These values are limited by the dead time and by the efficiency of the single-photon detectors used in the current experiment (20 $\mu \mathrm{s}$ dead time and $20 \%$ efficiency). We expect to achieve higher rates with faster detectors, such as superconductive nanowire single-photon detectors (SNSPDs) with very high efficiency $(80 \%)$, low dark counts (around $100 \mathrm{~Hz}$ ) and low dead time $(20$ ns), i.e. a sifted key rate of $1.5 \mathrm{MHz}$ can be obtained with such detectors. Additionally, the superposition states in the OAM fiber slowly drift due to temperature and geometric perturbations, so that implementing a phase-locked loop monitoring the phase through the fiber might help to increase stability, as shown in [15]. However, in the current setup, without any additional stabilization loops, a successful long term measurement was conducted for about 2 hours for the $\mathcal{M}_{0}$ basis, as shown in Supp. Mat.. Contrary to most other Hi-D setups reported to date, where only one quantum state can be detected at a time, our optical receiver design enables a higher detection efficiency and secret key generation rate [7, 21]. Moreover, in principle it allows a complete device-independent demonstration and loopholes free measurements for non-locality tests [38, 39]. Indeed, for a $D$ dimensional Hilbert space detection loophole free test, $D+1$ outcomes are required to strictly violate Bell's inequalities. However, projecting on $N<D$ outcomes, only a subset of all emitted pairs are measured, introducing possible classical correlation [16, 40]. 


\section{Conclusions}

In this work, we propose and demonstrate the use of twisted photons for fiber based high-dimensional quantum communication. We successfully prove the principle by sending twoand four-dimensional MUBs through $1.2 \mathrm{~km}$ of a special aircore fiber. Superposition states of different OAM modes have been generated, transmitted and detected in the experiment. The QBERs and secret key rates confirm the correct transmission and interpretation of the QKD schemes. These results represent a key point for the development of distributed quantum applications, proving that fiber based spatial modes protocols can be used for quantum communications. This experiment paves the way for future investigation on distributed high-dimensional entangled states and on fundamental properties of quantum mechanics, using OAM fibers.

\section{Acknowledgements}

We thank C. C. W. Lim, S. Paesani, J. Cotler, D. G. Marangon, P. Gregg and G. Carvacho for the fruitful discussions. This work is supported by the Center of Excellence, SPOC-Silicon Photonics for Optical Communications (ref DNRF123), by the People Programme (Marie Curie Actions) of the European Union's Seventh Framework Programme (FP7/20072013) under REA grant agreement $n^{\circ} 609405$ (COFUNDPostdocDTU) and by the Office of Naval Research MURI program (N00014-13-1-0627) and the National Science Foundation (ECCS-1610190).

\section{Author contributions}

D. Bacco conceived the experiment. D. Cozzolino, D. Bacco, B. Da Lio performed the system experiment. D. Bacco and K. Dalgaard designed the electrical controlling circuits. D. Bacco, B Da Lio, D. Cozzolino carried out the theoretical analysis on the proposed protocol. D. Cozzolino, D. Bacco, B. Da Lio, S. Ramachandran, M. Galili , K. Rottwitt, and L. K. Oxenløwe discussed the results. All authors contributed to the writing of the manuscript.

\section{Competing financial interests}

The authors declare no competing financial interests.

\section{Supplementary materials}

Supplementary materials are available under request.

\section{References}

[1] B. Fröhlich, et al., A quantum access network. Nature, 501(7465) (2013).

[2] M. Erhard, et al., Twisted photons: new quantum perspectives in high dimensions, Light: Science \& Applications 7.3 (2018).
[3] B. Fröhlich, et al., Long-distance quantum key distribution secure against coherent attacks, Optica 4 (2017).

[4] B. Korzh, et al., Provably secure and practical quantum key distribution over $307 \mathrm{~km}$ of optical fiber, Nat. Photon. 9 (163) (2015).

[5] J. G. Ren, et al., Ground-to-satellite quantum teleportation. Nature 549 (70) (2017).

[6] J. Yin, et al., Satellite-based entanglement distribution over 1200 kilometers, Science 356 (1140) (2017).

[7] F. Bouchard, et al.,Underwater Quantum Key Distribution in Outdoor Conditions with Twisted Photons, arXiv 1801.10299v1 (2018).

[8] J. Ling, et al., Towards quantum communications in free-space seawater, Opt. Express 25 (2017).

[9] N. Cerf, et al., Security of Quantum Key Distribution Using d -Level Systems Phys. Rev Lett., 88 (127902) (2002).

[10] F. Bouchard, et al., High-dimensional quantum cloning and applications to quantum hacking, Sci. Adv., 3 (2017).

[11] N. T. Islam, et al., Provably secure and high-rate quantum key distribution with time-bin qudits, Sci. Adv., 3 (2017).

[12] Y. Ding, et al., High-Dimensional Quantum Key Distribution based on Multicore Fiber using Silicon Photonic Integrated Circuits, npj Quantum Information 3:25 (2017).

[13] D. Bacco, et al., Two-dimensional distributed-phasereference protocol for quantum key distribution, Scientific Reports 6: 36756 (2016).

[14] D. Bacco, et al., Space division multiplexing chip-to-chip quantum key distribution, Scientific Reports 7: 12459 (2017).

[15] G. Cañas,et al., High-dimensional decoy-state quantum key distribution over multicore telecommunication fibers, Phys. Rev. A, 96, 022317 (2017).

[16] J. Wang, et al., Multidimensional quantum entanglement with large-scale integrated optics, Science 36, 8075 (2018).

[17] N. Bozinovic, et al., Terabit-scale orbital angular momentum mode division multiplexing in fibers, science 340.6140 (2013).

[18] C. Brunet, et al., Design, fabrication and validation of an OAM fiber supporting 36 states, Optics express 22.21 (2014).

[19] M. Krenn, et al., Twisted light transmission over $143 \mathrm{~km}$, Proceedings of the National Academy of Sciences 113 (48) (2016). 
[20] M. Mirhosseini, et al., High-dimensional quantum cryptography with twisted light, New Journal of Physics, 17 (2015).

[21] A. Sit, High-dimensional intracity quantum cryptography with structured photons, Optica 4 (2017).

[22] S. Gröblacher, et al. Experimental quantum cryptography with qutrits, New Journal of Physics, 8 (2006).

[23] S. Etcheverry, et al., Quantum key distribution session with 16-dimensional photonic states, Scientific reports 3 (2013).

[24] G. Vallone, et al., Free-space quantum key distribution by rotation-invariant twisted photons, Physical review letters 113 (2014).

[25] N. Bozinovic, et al., Record-length transmission of photons entangled in orbital angular momentum (OAM), Frontiers in Optics 2011/Laser Science XXVII, OSA Technical Digest, paper PDPB1 (2011).

[26] Jian Wang, et al., Terabit free-space data transmission employing orbital angular momentum multiplexing, Nature photonics 6 (2012).

[27] L. Hoi-Kwong, et al., Decoy State Quantum Key Distribution, Physical review Letter 94 (2005)

[28] L. Marrucci, et al., Optical spin-to-orbital angular momentum conversion in inhomogeneous anisotropic media., Physical review letters 96.16 (2006)

[29] P. Gregg, et al., Conservation of orbital angular momentum in air-core optical fibers, Optica 2 (2015).

[30] J. Leach, et al., Measuring the orbital angular momentum of a single photon, Physical review letters $\mathbf{8 8 . 2 5}$ (2002).

[31] M. J. Padgett, et al., Dove prisms and polarized light, Journal of Modern Optics 46 (1999).

[32] R. Saad, et al., Highly selective 7 orbital angular momentum mode multiplexer based on multi-plane light conversion, in Proc. ECOC, P1.SC1.15 (2017).

[33] M. N. O'Sullivan, et al., Near-perfect sorting of orbital angular momentum and angular position states of light, Optics express 20.22 (2012).

[34] C. H. Bennett, G. Brassard, Quantum Cryptography: public key distribution and coin tossing, in Proceeding of IEEE International Conference on Computer, Systems $\mathcal{E}$ Signal Processing, 175-179 (1984).

[35] V. Scarani, et al., The security of practical quantum key distribution. Reviews of Modern Physics, 81(3), 1301-1350 (2009)
[36] X. Ma, et al., Practical decoy state for quantum key distribution, Phys. Rev. A, 72(1), 012326 (2005).

[37] N. T. Islam, et al., Securing quantum key distribution systems using fewer states, arXiv:1801.03202, (2018).

[38] G. Weihs, Violation of Bell's Inequality under Strict Einstein Locality Conditions, Phys. Rev. Lett., 81 (1998)

[39] B. Cyril, et al., Detection loophole in Bell experiments: How postselection modifies the requirements to observe nonlocality, Phys. Rev. A, 83, 032123 (2011)

[40] M. Kues, et al., On-chip generation of high-dimensional entangled quantum states and their coherent control, Nature 546 622-626 (2017)

[41] D. J. Richardson, et al., Space-division multiplexing in optical fibers, Nature Photonics 7 (2013). 